\title{
Topological surface integrity modification of AISI 1038 alloy after vibration-assisted ball burnishing
}

\author{
R. Jerez-Mesa ${ }^{\mathrm{a}, *}$, Y. Landon ${ }^{\mathrm{a}}$, J.A. Travieso-Rodriguez ${ }^{\mathrm{b}}$, G. Dessein ${ }^{\mathrm{c}}$, J. Lluma-Fuentes ${ }^{\mathrm{d}}$, V. Wagner ${ }^{\mathrm{c}}$ \\ a Institut Clément Ader, Université de Toulouse III Paul Sabatier, 3 Rue Caroline Aigle, 31400 Toulouse, France \\ b Mechanical Engineering Department, Universitat Politècnica de Catalunya, Av. Eduard Maristany, 10-14, 08019 Barcelona, Spain \\ ${ }^{\mathrm{c}}$ Laboratoire Génie de Production, École Nationale d'Ingénieurs de Tarbes, 47 Avenue d'Azereix, 65000 Tarbes, France \\ ${ }^{\mathrm{d}}$ Material Science and Metallurgical Engineering Department, Universitat Politècnica de Catalunya, Av. Eduard Maristany, 10-14, 08019 Barcelona, Spain
}

\section{ARTICLE INFO}

\section{Keywords:}

Vibration-assisted ball burnishing

Surface topology

Surface texture

Roughness

Abbott-firestone

\begin{abstract}
A B S T R A C T
The objective of this paper is to analyze the effect of the vibration-assisted ball burnishing process on the topology of AISI 1038 flat surfaces, in order to evaluate its feasibility for surface enhancement towards wear prevention and fatigue enhancement in industrial components. With that aim, an experimental campaign based on a Taguchi orthogonal matrix has been deployed. Five factors were studied, namely: preload force, number of passes, feed, initial surface texture and strategy. The topologies of the resulting burnishing patches have been acquired with a non-contact optical device, and the 3D texture parameters have been calculated to quantify the effects of burnishing. In all cases, the bearing capacity of the burnished surfaces was improved, as the proportion of core material is increased due to the deformation of the surface peaks. The initial surface state proved to be the most influential parameter on amplitude, spatial, and volumetric parameters. In all cases, a set of optimal vibration-assisted ball burnishing parameters was found for the sake of reproducibility and systematization of the process. Finally, results have been compared to the conventional ball burnishing process, observing that it presents scratch damage on the surfaces that can be prevented through assistance through vibrations.
\end{abstract}

\section{Introduction}

Ball burnishing is a mechanical finishing operation, performed on engineering parts to improve their surface integrity, in search of enhanced performance in highly demanding regimes. The process is strictly based on local plastic deformation of the surface irregularities by the action of a spherical indenter, which rolls over it while transmitting a calibrated precharged force [1].

Results of ball burnishing on steel alloys as a means of enhancing the surface integrity have been extensively tackled in the bibliography. Surface integrity is understood as a comprehensive concept including numerous parameters, which describe the state of a surface after being subjected to a specific manufacturing process. The scientific literature deals specifically with the results of ball burnishing focus on three specific components of surface integrity, namely, roughness, residual stress and hardness.
The effectiveness of ball burnishing to reduce the surface roughness of steel parts has been confirmed in flat surfaces. Shiou \& Cheng (2008) [2] proved that burnishing on NAK 80 steel moulds achieved up to $90 \%$ improvement. Travieso-Rodríguez et al. (2011) [3] also observed similar improvement rates on AISI 1038 concave and convex surfaces. Positive results on the increase of surface hardness due to strain hardening, were demonstrated by Loh et al. (1990) [4], showing an increase by $55 \%$ after ball burnishing of a medium carbon steel alloy. Avilés et al. (2013) [5] confirmed the increase of fatigue life due to higher compressive residual stress after ball burnishing. The layer thickness of material affected by the process can reach $500 \mu \mathrm{m}$. This deep strain hardening of metals due to burnishing can be explained by Herzian contact stress propagation mechanics. Rodríguez et al. (2012) [6] identified crack nucleation and propagation in cylindrical AISI 1045 specimens due to a higher and deeper compressive residual stress obtained through ball burnishing. For all those reasons, burnishing is cited as an inexpensive method to attain a comprehensive and full enhancement of surface integrity [7].

\footnotetext{
* Corresponding author. (G. Dessein); jordi.lluma@upc.edu (J. Lluma-Fuentes); vincent.wagner@enit.fr (V. Wagner)

Email addresses: ramon.jerez-mesa@univ-tlse3.fr (R. Jerez-Mesa); yann.landon@univ-tlse3.fr (Y. Landon); antonio.travieso@upc.edu (J.A. Travieso-Rodriguez); gilles.dessein@enit.fr 
Among the multiple parameters describing surface integrity, this paper focuses on texture parameters. Extensive work exists analyzing the surface topography after burnishing, establishing that results are mainly dependent on the applied force [8] and the initial roughness state [9]. Authors have conventionally chosen 2D surface roughness parameters, mainly the average surface roughness $R_{a}$, to describe the surface state $[10,11]$. In some cases, extreme points such as $R_{t}$ or $R_{z}[12]$ have been used to evaluate burnishing results.

In this paper, an alternative method to approach surface topology is proposed to evaluate ball burnishing results. First of all, because the average surface roughness is actually an obsolete parameter with no statistical meaning, and should be substituted by the RMS value of heights, or $\mathrm{R}_{q}$, as pointed out by Sherrington et al. (1987) [13]. Secondly, leaping from $2 \mathrm{D}$ to $3 \mathrm{D}$ parameters to analyze the results of ball burnishing is expected to reveal more comprehensive information about the surface state. With that objective, burnishing patches shall be acquired, and the 3D texture parameters defined in the ISO 25178 standard, namely, the $12-\mathrm{S}$ and $13-\mathrm{V}$ sets, shall be calculated and compared [14].

Moreover, an innovative modification of the conventional ball burnishing process is featured in this paper, by introducing a high frequency oscillatory component to the burnishing preload force. This process is called vibration-assisted ball burnishing (VABB), and has barely been explored so far in the scientific literature. The physical phenomena on which VABB is based is called acoustoplasticity, that is, the overall reduction of the quasi-static stress required to deform a material as a result of the superimposition of vibrations on the deforming force [15].

The affectation of materials due to acoustoplasticity varies according to their mechanical properties, but, in general, there is a direct relationship between acoustic impedance and the degree to which the material is affected by the effect [16]. On the contrary, the acoustoplastic effect is not strictly related to ultrasonic frequency levels, as prove the works developed by Kirchner et al. (1985) [17]. Indeed, favorable results due to the application of acoustoplasticity to ball burnishing using low and medium-frequency vibrations can be found in the literature. For instance, Pande \& Patel (1983) [18] proved that a $60-\mathrm{Hz}$ vibratory force allows increases surface hardness at $40 \%$ in a mild steel alloy.

Medium frequency VABB was tested by Gómez-Gras et al. (2015) [19], who designed a VABB tool assisted by $2.1 \mathrm{kHz}$ vibrations. The alternative force was originated by the alternative deflection of thin plates by means of a coil excited by an alternate electrical field. The tool proved to deliver positive results in terms of surface roughness on G10380 steel [20] and A92017-T4 specimens [21], by achieving in both materials the same $R_{a}$ and $R_{t}$ reduction results with one pass of the VABB process and with 5 passes of the non-vibration-assisted ball burnishing (NVABB) one.

40-kHz assisted ball burnishing has been also tested on several materials, but only at a preliminary level. Ti-6Al-4V flat surfaces were treated by Bozdana et al. (2005) [22] with a hydrostatic burnishing tool, achieving a minimum $R_{a}$ of $0.074 \mu \mathrm{m}$ and $0.168 \mu \mathrm{m}$ through the NVABB and VABB processes, respectively. Although average roughness was slightly higher for VABB, it was obtained with $160-\mathrm{N}$ static force, whereas the $1160-\mathrm{N}$ preload was used at the NVABB process. These results were later confirmed by the same authors, who also showed the higher effectiveness of VABB at increasing residual stress on the titanium specimens [23]. Huuki et al. (2013) [24] proved the positive effect of VABB on the surface roughness, out-of-roundness, and hardness and the diameter of turned 34CrNiMo6-M tempering steel, and, later, on AW6082-T6 aluminum and S355J2 structural steel specimens [25].

The bibliographical review exhibits the lack of references reporting a detailed analysis of 3D parameters calculation to evaluate the results of the ball burnishing operation. Secondly, no in-depth comprehensive studies about the VABB process can be found. To fill in this niche, this paper presents a new insight in the topographical analysis of VABB re- sults. To that aim, a design of experiments (DOE) based on Taguchi arrays is applied. As topography determines important in-service properties of engineering parts, such as wear, lubricant retention or debris evacuation, characterizing the texture features achieved after VABB is necessary to valorize the process industrially and being able to proceed to its implementation in production environments.

\section{Materials and methods}

\subsection{Specimen preparation}

The surface of a $100 \times 200 \mathrm{~mm}$ block cut from an AISI 1038 laminated bar was used to perform all burnishing tests. Fig. 1 shows a schema of the workpiece and the burnishing context. The specimen was surfaced with a $\emptyset 80$ mill with 5 cutting inserts. On the flat surface, three texture zones $\left(\mathrm{S}_{1}^{0}, \mathrm{~S}_{2}^{0}\right.$ and $\left.\mathrm{S}_{3}^{0}\right)$ were machined through parallel passes with a $\varnothing 10 \mathrm{TiN}$ coated hemispherical milling tool. To generate three different textures, the offset between passes $a_{e}$ was increased from 0.5 to 1.1 in steps of $0.3 \mathrm{~mm}$. In all cases, a $0.2 \mathrm{~mm}$ depth of cut was used. As shall be explained below, the three different finishing zones are included in the DOE as a factor. This finishing strategy was chosen because it is highly extended in the manufacturing industry to finish flat, concave and convex parts through five-axis routines, following trajectories automatically generated by CAM software. Hence the industrial interest in studying this kind of surfaces.

\section{2. $V A B B$ tool and experimental setup}

The self-designed burnishing prototype used in this paper (Fig. 2) is composed of three main parts, as explained in depth by Jerez-Mesa et al. (2018) [26], which can be described as follows:

- Force regulation module. The preload force is regulated by means of a spring which is compressed on the surface during the prepara-

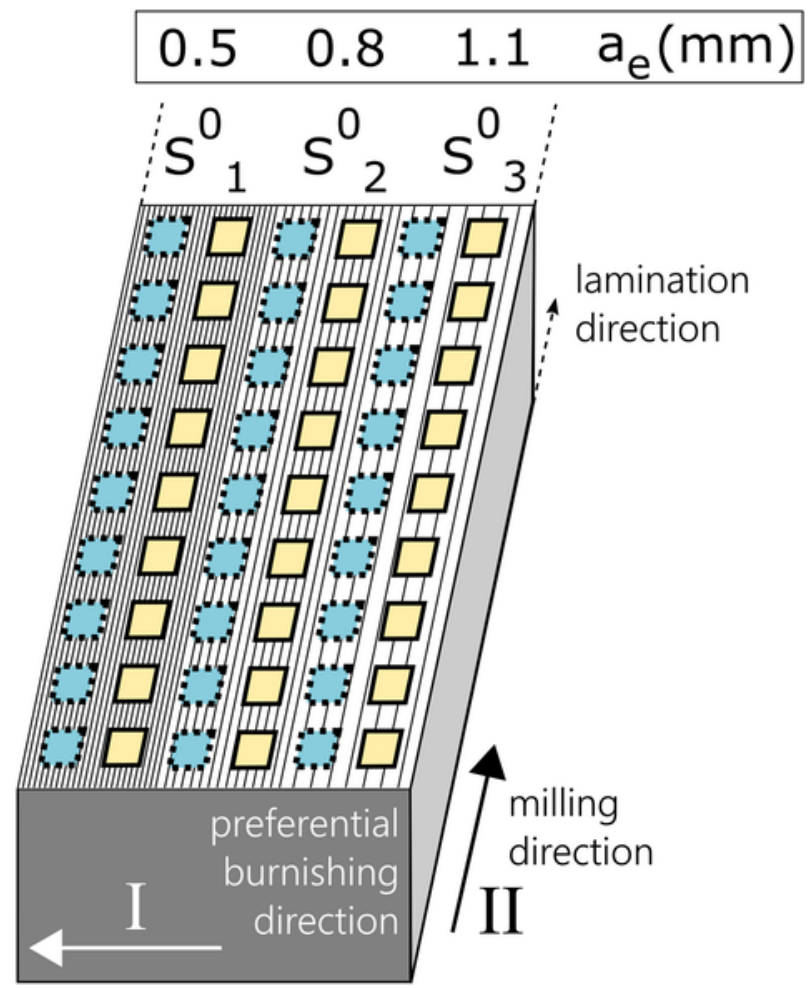

Fig. 1. Overview of burnishing workpiece with different initial surface states, and nomenclature of directions used in this paper. Continuous line patches: NVABB. Discontinuous line patches: VABB. 


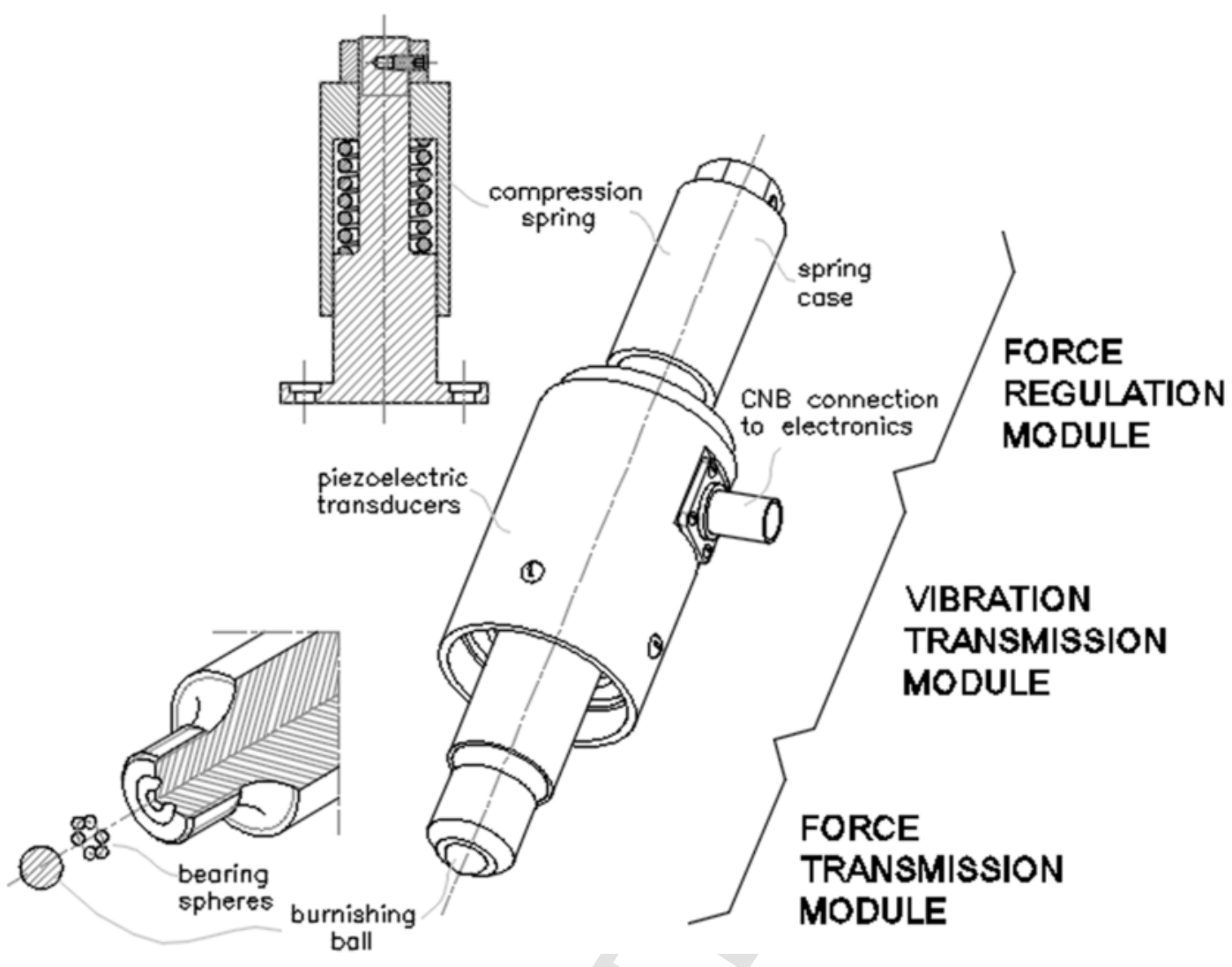

Fig. 2. Schematic representation of the prototype and its modules, with details of the force regulation and transmission modules.

tory phase of the burnishing process. As Hooke's law governs the force provided by the spring, its elastic constant and precompression force must be calibrated during a preparatory phase, by using a force table. The used spring is characterized by a stiffness $k$ of $83.4 \mathrm{~N} / \mathrm{mm}$. The spring is housed inside a cylinder, which serves also as the element clamped by the tool holder to attach the tool to the milling machine.

- Vibrations transmission module. This unit is composed by a stacked piezoelectric transducer contained inside a case. To perform the VABB operation, the piezoelectric is excited by the external circuit with a $40 \mathrm{kHz}$ frequency electrical signal. It is also designed so that the ball free displacement is of around $10 \mu \mathrm{m}$. When the piezoelectric is not excited, the prototype can be used as a NVABB tool. To check these design specifications, a preliminary experimental phase in two phases using an acoustic emission sensor and a dynamometer, both connected to a 10-MHz sampling device, are performed. All signals acquired for several preloads derive in a uniform $38.09 \mathrm{kHz}$ signal, thus confirming that the process is stable. A second phase of dynamometric measurements allowed to quantify the oscillating force according to a sinus wave of $2.84 \pm 0.048 \mathrm{~N}$ amplitude, confirming the previously observed frequency.

- Force transmission module. The force is transmitted to the objective material by means of a ball supported on a set of bearing spheres inside a hemispherical slot machined in the tool head. That head is coupled to the base where the previous module is mounted, so that the vibratory movement of the piezoelectric is transmitted to the ball while it rolls on the surface during the process.

Tests were performed in a HURON KX 10 HSM milling machine. To regulate the burnishing preload and record the burnishing force along the different burnishing experiments, the workpiece was mounted on the machine through screws on a Kistler 9171A dynamometric table. The preload was kept inside a controlled span during all tests.

\subsection{Taguchi design of experiments}

The design of experiments approach constitutes one of the most used techniques to perform an in-depth assessment of engineering case studies, because it allows to evaluate the influence of different factors at different levels on one or more response variables. For that reason, it has been extensively used to study the influence of the burnishing factors on the texture after burnishing of different materials [27-29]. For this experimentation, the selected factors and levels are shown in Table 1, and can be explained as follows.

- Preload force. It is the static component of the burnishing force, and is determined by the spring compression length. The vibratory force is overlapped on this force, resulting on the total burnishing force.

- Number of passes. Amount of successive iterations performed during the burnishing operation to cover the whole surface. The way these passes are overlapped and executed is described through the Strategy parameter, explained at the last item.

- Feed. Linear velocity at which the tool is translated over the surface. It is easily commanded through the NC code by means of the F function. Therefore, it corresponds to the conventional feed in a milling operation, with $\mathrm{mm} / \mathrm{min}$ units.

Table 1

Factors and levels included in the Taguchi DOE.

\begin{tabular}{lll}
\hline Factor & Symbol & Levels \\
\hline Preload (N) & $F_{p}$ & $90-180-270$ \\
Number of burnishing passes & $n_{p}$ & $1-3-5$ \\
Feed (mm/min) & $v_{f}$ & $300-600-900$ \\
Initial surface state & $\mathrm{S}^{0}$ & $\mathrm{~S}^{0}{ }_{1}-\mathrm{S}^{0}{ }_{2}-\mathrm{S}^{0}{ }_{3}$ \\
Strategy & St. & bn0-bn90/0-nb0 \\
\hline
\end{tabular}


- Starting surface topology. The influence of the departing surface on the burnishing results is of high interest, in order to associate the effectiveness of the process with a certain finishing degree. To that purpose, three different surfaces were included in the model, as was represented above.

- Strategy. This parameter describes the trajectory followed by the burnishing ball to span the whole burnishing patch. The strategy is executed according to successive linear interpolations defined in the NC code. Fig. 3 represents the three chosen strategies, which are coded according to a possible change of direction of even passes, and to the order in which those passes are developed. All three strategies perform the first pass along direction I, a.k.a. preferential burnishing direction, perpendicular to the initial milling direction II. Only the strategy nb90/0 contemplates a change in direction, so that even passes are performed along direction II.

To implement the experimental study, an L27 ( $\left.3^{13}\right)$ Taguchi orthogonal array was chosen. This configuration allows to analyze up to 13 factors at 3 levels. However, as only 5 factors were included in the model, the number of degrees of freedom provided by the array enabled to evaluate the influence of three of the possible interactions, as represented through the linear graph included at Fig. 4. To avoid aliasing effects among factors, these were located in the Taguchi array at the columns indicated at the linear graph. This procedure is standardized, and can be consulted at Taguchi et al. (2005) [30]. The final experimental array is represented at Table 2 . To make easier the later interpretation of results, the runs with the three different forces are shadowed in the table.

\subsection{Texture measurement}

\subsubsection{Topological parameters}

The surface quality state has been traditionally described in manufacturing engineering through the $R_{a}$ and the $R_{t}$ parameters. This paper offers a new insight on topological analysis of the burnishing process by calculating the tridimensional S-surface- and V-volumetric-sets of parameters, expected to provide further information with regards to the one exhibited by two-dimensional $R_{a}$ and $R_{t}$

The $S$ parameters are extensively explained in Dong et al. (1992, 1993, 1994a, 1994b) [31-34]. They can be divided in four main groups, which can be explained as follows:

- Amplitude parameters. These parameters represent statistical characteristics of the surface height, extreme points, and the shape of the surface height distribution. The root-mean-square deviation $S_{q}$ is the RMS of the height distribution over and under the least-squares mean plane, and is eligible to substitute the average height $S_{a} . S_{10 z}$ represents the amplitude of maximum deviations. Skewness $S_{\text {sk }}$ and Kurtosis $S_{k u}$ are the third and fourth moments of the height distribu- tion histogram, and are used to characterize the relative weight of negative and positive heights in the surface, thus enabling to describe its shape. More specifically, positive values of $S_{s k}$ represent surfaces with prevailing valleys, whereas negatively skewed surfaces are mainly composed of positive heights. As for $S_{k w}$, values higher than 3 represent abrupt surfaces. On the contrary, values lower than 3 represent smoother surfaces.

- Spatial parameters. These descriptors introduce the factor of directionality in the characterization of the surface. They are calculated according to the autocorrelation function of the sampled surface, AACF, which describes the dependence of the height data at one position on the values at another position of the same dataset. $S_{a l}$ represents the shortest distance in which the value of the AACF has decayed under a threshold, normally 0.2 . Large values of this parameter represent surfaces dominated by low frequency components, that is, the surface features are repeated along longer distances. On the other hand, $S_{t r}$ allows to identify the existence of a preferential texture pattern. This ratio ranges between 0 and 1 , being 0 for surfaces who show strong anisotropy, that is, with an outstanding preferential direction in the texture.

- Hybrid parameters. They combine spatial and amplitude features of the surface, by evaluating the degree of heterogeneity of its average slope. $S_{d r}$ represents the ratio of the increment of interfacial area of a surface over the flat sampling area. Values of the developed interfacial area lower than $1 \%$ are usually associated to superfinished surfaces. Additionally, $S_{d q}$ calculates the root mean square of the surface slope within the sampling area, so that a higher value indicates a rougher surface. Although it is not so intuitive as the $S_{d r}$ parameter, lower $S_{d q}$ values are associated to surface textures nearer to finishing state.

- Miscellaneous parameter. The $S_{t d}$ parameter is the angle value that indicates the direction of the main surface features in anisotropic surfaces.

On the other hand, the $\mathrm{V}$ parameter set $\left(V_{m p}, V_{m c}, V_{v c}, V_{v v}\right)$ describe the bearing curve of the height distribution, and, therefore, allow to adopt a functional perspective of the height distribution of a surface, as represented in Fig. 5. This curve was conceived by Abbott and Firestone (1933) [35], and represents the accumulated height distribution of a surface. To make this curve profitable, the ISO 13565-2 describes the method through which the peak, valley and core material volumes are established. The method consists on tracing the minimum-slope secant line, spanning $40 \%$ of the bearing area. The intersection with the curve, defines the boundaries between the aforementioned portions: $V_{m p}$ and $V_{m c}$ respectively represent the amount of material in the peak and core portions, whereas $V_{v c}$ and $V_{v v}$ reflect the void volume in the core and the valley portions. Additionally, it allows to calculate a three-descriptor set that describe the geometry of the bearing area curve, namely $S_{p k}$ (height of the peak portion), $S_{k}$ (height of the core
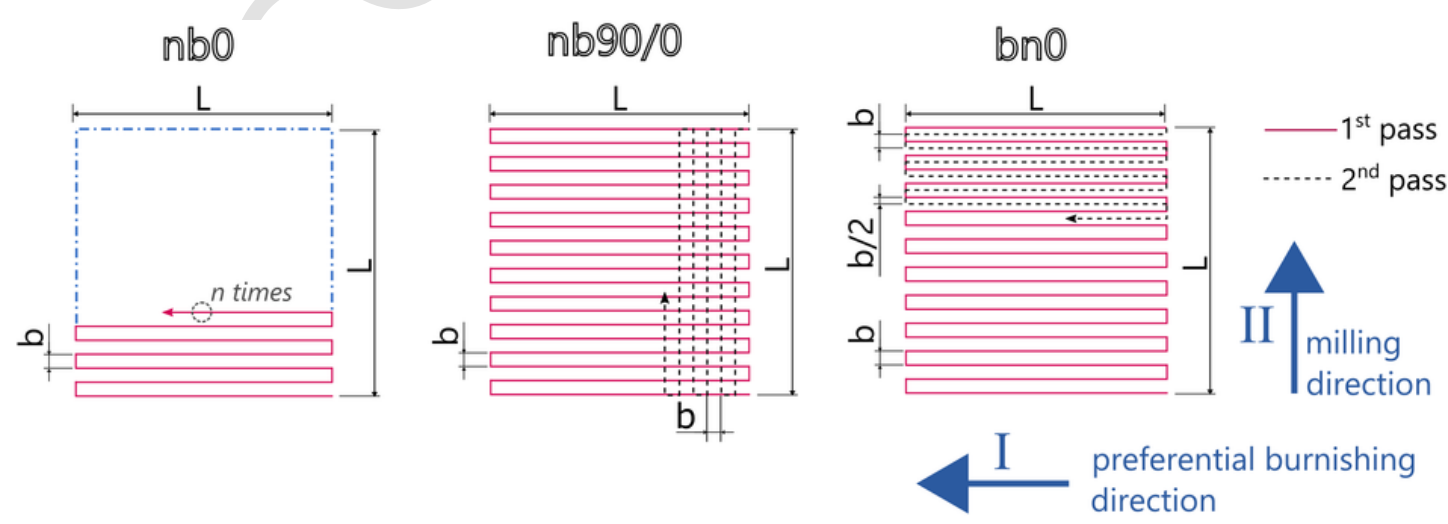

Fig. 3. Burnishing strategies included in the DOE and nomenclature of the directions. 


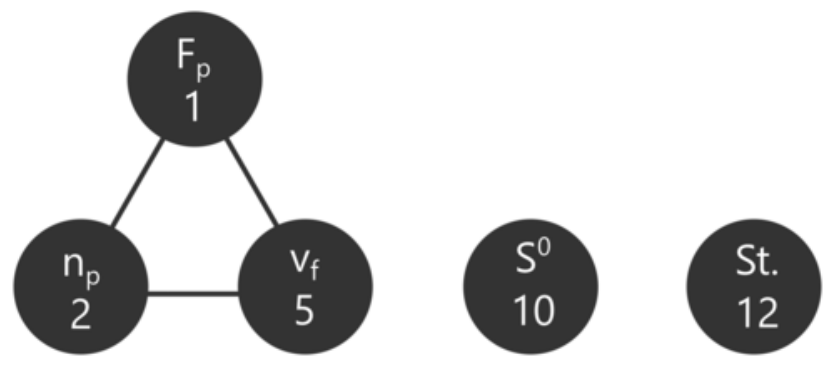

Fig. 4. Linear graph representing the factors (spheres) and interactions (lines), along with their column in the Taguchi array.

portion) and $S_{v k}$ (depth of the valley portion). Essentially, the material above the core is associated to the material which shall eventually experience running-in wear. The core corresponds to the stationary bearing material, and the valley zone is related to the surface capability for lubricant retention and debris evacuation.

\subsubsection{Equipment and sampling strategy}

To calculate the specified parameters, the height distribution of every patch derived from all burnishing tests were acquired by an ALICONA Infinite Focus Microscope equipped with a $10 \times$ objective. In order to discard edge effects, only the central area of each burnishing patch was acquired. The system functions based on the focus variation technology, that is, by the acquisition of the height of all points composing the target surface by an optical head that takes images along a vertical $100-\mathrm{nm}$ resolution. A $0.1-\mu \mathrm{m}$ sampling interval was used both in $x$ and $y$ directions. The size of each patch is described in the following subsection, as it varies for each of the three initial tested areas.

The filtering of the dataset and the calculation of parameters were performed by the ALICONA IF MeasureSuite software. The dataset was subjected to an automatic filtering technique based on the repeatability to discard outliers in it. Then, a Gaussian low-frequency filter was applied to retain the texture profile, equivalent to the two-dimensional $\mathrm{P}$ profile, according to the ISO 11562 . Like that, all S and V parameters shall refer to texture data. Specific measurements concerning roughness parameters shall be calculated on the raw profile, by applying a

Table 2

Experimental plan based on the $\mathrm{L}^{27}$ Taguchi array.

\begin{tabular}{|c|c|c|c|c|c|}
\hline run & $F_{p}$ & $n_{p}$ & $v_{f}$ & $\mathrm{~S}_{0}$ & St. \\
\hline 1 & 90 & 1 & 300 & 1 & nb0 \\
\hline 2 & 90 & 1 & 600 & 2 & bn $90 / 0$ \\
\hline 3 & 90 & 1 & 900 & 3 & bn0 \\
\hline 4 & 90 & 3 & 300 & 2 & bn0 \\
\hline 5 & 90 & 3 & 600 & 3 & nb0 \\
\hline 6 & 90 & 3 & 900 & 1 & bn $90 / 0$ \\
\hline 7 & 90 & 5 & 300 & 3 & bn $90 / 0$ \\
\hline 8 & 90 & 5 & 600 & 1 & bn0 \\
\hline 9 & 90 & 5 & 900 & 2 & nb0 \\
\hline 10 & 180 & 1 & 300 & 3 & bn $90 / 0$ \\
\hline 11 & 180 & 1 & 600 & 1 & bno \\
\hline 12 & 180 & 1 & 900 & 2 & nb0 \\
\hline 13 & 180 & 3 & 300 & 1 & nb0 \\
\hline 14 & 180 & 3 & 600 & 2 & bn $90 / 0$ \\
\hline 15 & 180 & 3 & 900 & 3 & bn0 \\
\hline 16 & 180 & 5 & 300 & 2 & bn0 \\
\hline 17 & 180 & 5 & 600 & 3 & nb0 \\
\hline 18 & 180 & 5 & 900 & 1 & bn $90 / 0$ \\
\hline 19 & 270 & 1 & 300 & 2 & bn0 \\
\hline 20 & 270 & 1 & 600 & 3 & nb0 \\
\hline 21 & 270 & 1 & 900 & 1 & bn $90 / 0$ \\
\hline 22 & 270 & 3 & 300 & 3 & bn $90 / 0$ \\
\hline 23 & 270 & 3 & 600 & 1 & bn0 \\
\hline 24 & 270 & 3 & 900 & 2 & nb0 \\
\hline 25 & 270 & 5 & 300 & 1 & $\mathrm{nb} 0$ \\
\hline 26 & 270 & 5 & 600 & 2 & bn $90 / 0$ \\
\hline 27 & 270 & 5 & 900 & 3 & bn0 \\
\hline
\end{tabular}
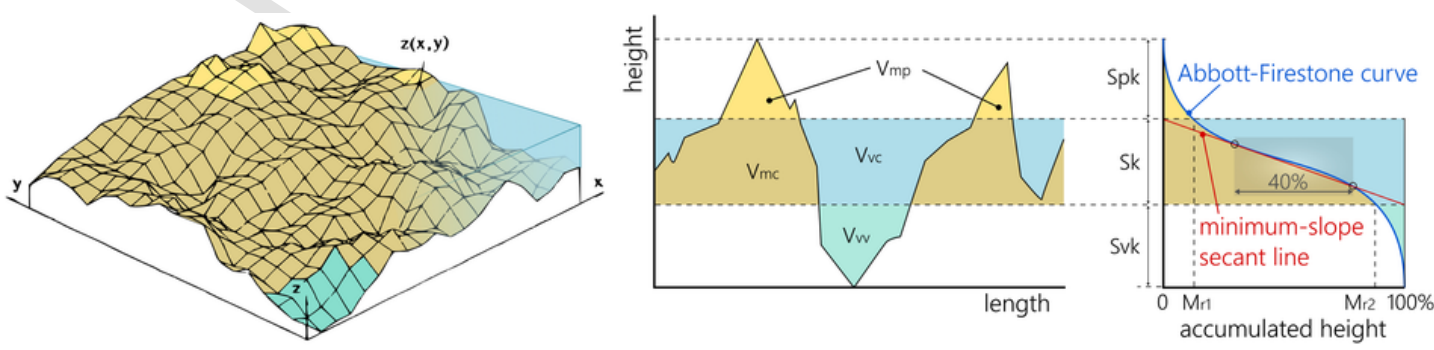

Fig. 5. V parameters related to the bearing area curve, represented in $2 \mathrm{D}$ and $3 \mathrm{D}$. 
Gaussian digital filter with a cut-off length of $0.25 \mu \mathrm{m}$ and an evaluation length of $1.25 \mathrm{~mm}$.

\subsubsection{Determination of the acquired patch size}

To determine the size of each measurement, a preliminary study was performed, thus finding the optimal dimensions enabling to find representative topological information while minimizing the acquisition time. With that aim, the topologies of the three initial machined surfaces were registered according to patches of increasing dimensions. As the three machined topologies consist on a periodic wavy pattern formed by alternated peaks and valleys, it was decided to adjust the dimensions of each patch to an integer value of a coupled peak + valley set (PV).

Fig. 6 shows the variation of the most relevant topological parameters with regards to the patch size. Kurtosis and skewness were essentially constant for all patches, whereas $S_{10 z}$ did not show any signal of stabilization. That is logical due to the way it is calculated, as pointed out by Dong et al. (1994a) [32], and, therefore, cannot be taken as a criteria to decide the patch size. However, $S_{q}$ proved to have a stable value for surface $S^{0}{ }_{1}$, while for the surface $S^{0}{ }_{3}$ required the acquisition
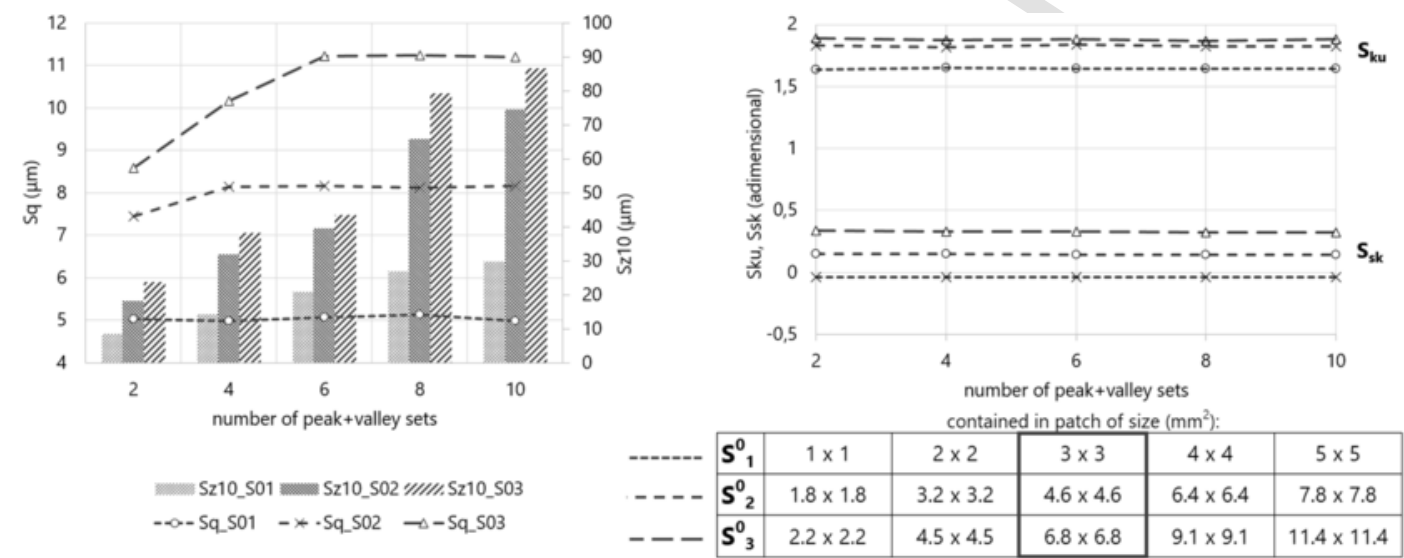

Fig. 6. Variation of amplitude descriptors of in function of the size of the acquired patches. Patch dimensions selected for each finishing zone are squared.
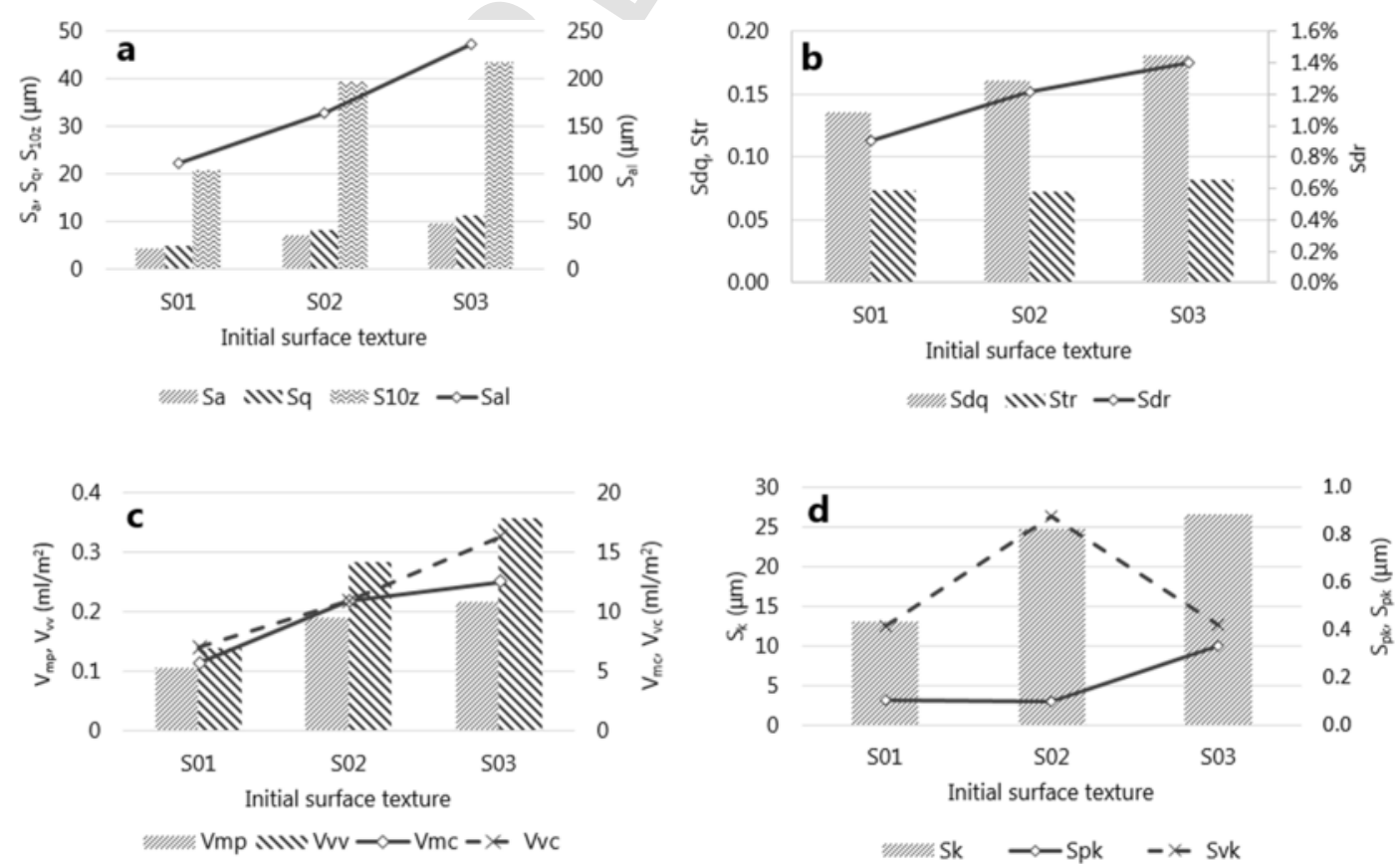

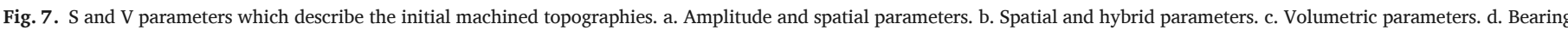
area curve descriptors. 
tion. Provided the statistical lack of meaning of $S_{a}$, only the $S_{q}$ parameter is to be considered from now on as the general indicator of surface amplitude.

As for the spatial parameters, $S_{a l}$ increases, which means that the characteristic length that defines a considerable change of the topological features of the surface is higher as the finishing zone is machined with a higher offset between passes. $S_{t r}$ is for the three cases well below 0.1 , and proves that all finishing machining strategies have lead to a highly anisotropic surfaces, with a preferential direction.

The $S_{d r}$ hybrid parameter shows that the three machined surfaces are next to a finishing state, although the $S^{0}{ }_{1}$ surface is the smoothest one. At sight of Fig. 7.b, the $S_{d q}$ shows the same trend, and, therefore, it is considered redundant in terms of results interpretation, and shall not be included in the analysis hereafter. The miscellaneous $S_{t d}$ parameter has not been included in the results, as it is 90 degrees for all surfaces, and does not provide substantial information.

Volumetric parameters also show the higher abruptness of the $\mathrm{S}_{3}^{0}$ with regards to the opposite $\mathrm{S}^{0}{ }_{1}$, as all core, peak and valley volumes, are maximum for $S_{3}^{0}$, and minimum for $S^{0}$, as was easy to predict (Fig. 7.c). The descriptors of the Abbott-Firestone curve show the same behavior with regards to the core and peak material at, but not so at the valley proportion (Fig. 7.d).

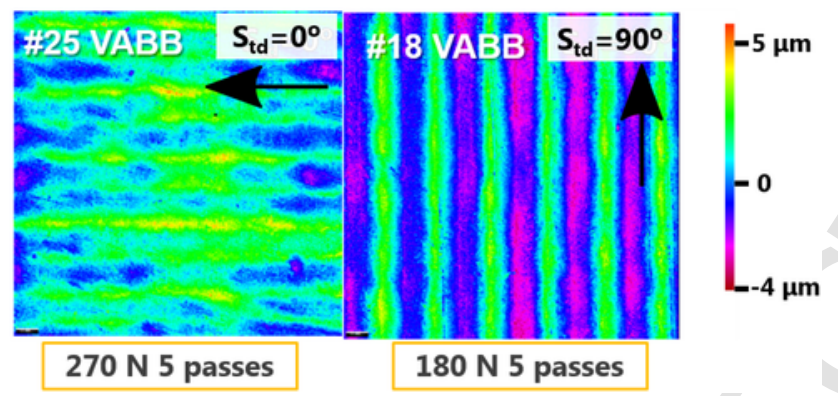

Fig. 8. Topological images of tests \#25 and \#18.

\section{2. $R$ parameters}

A preliminary visual inspection of the burnished surfaces was performed. Fig. 8 shows the topological color images of tests \#25 and \#18, performed on the $S^{0}{ }_{1}$ surface. It evidences a low expected repeatability of two-dimensional $R$ parameters to assess VABB results. For instance, test \#25 exhibits a higher heterogeneity of height distribution, compared to test \#18, which is clearly still driven by the initial machining marks. For this reason, all two-dimensional parameters were measured 20 times along the burnishing direction, and averaged to obtain a representative magnitude of these parameters.

The resulting $R_{q}$ value was subjected to an ANOVA. Fig. 9 shows the mean effects plot derived from that analysis. The VABB process proves to decrease the roughness, being the preload the most influential value, followed by the number of passes. The strategy and initial surface show no influence. Nor does the feed, at sight of the associated $p$-value calculated through the ANOVA test. No interactions are observed between $F_{p}$, $n_{p}$ and $v_{f}$. This result agrees with the previously analyzed bibliographical references.

\section{3. $S$ parameters}

\subsubsection{Amplitude parameters $S_{q}$ and $S_{10 z}$ : overall surface modification}

An ANOVA was performed taking the $S_{q}$ as response variable. Different results in terms of average influence are found, regarding $R_{q}$. Fig. 10 shows that the initial surface texture is the most influential parameter on the results, followed by the preload and the number of passes. The strategy and feed do not show a significant influence. On the other hand, no interaction between factors is detected as relevant in this case.

At sight of these ANOVA results, the topological parameters of the burnished surfaces have been grouped and represented according to their respective initial surface texture (Fig. 11). This layout reconfiguration of the experimental array allows to arrange the tests by increasing force, and by reiterative cycles of 1,3 and 5 passes. This simplification obviates the feed and the strategy, and enables to interpret the burnishing results as a discrete sequential evolution of the surface from
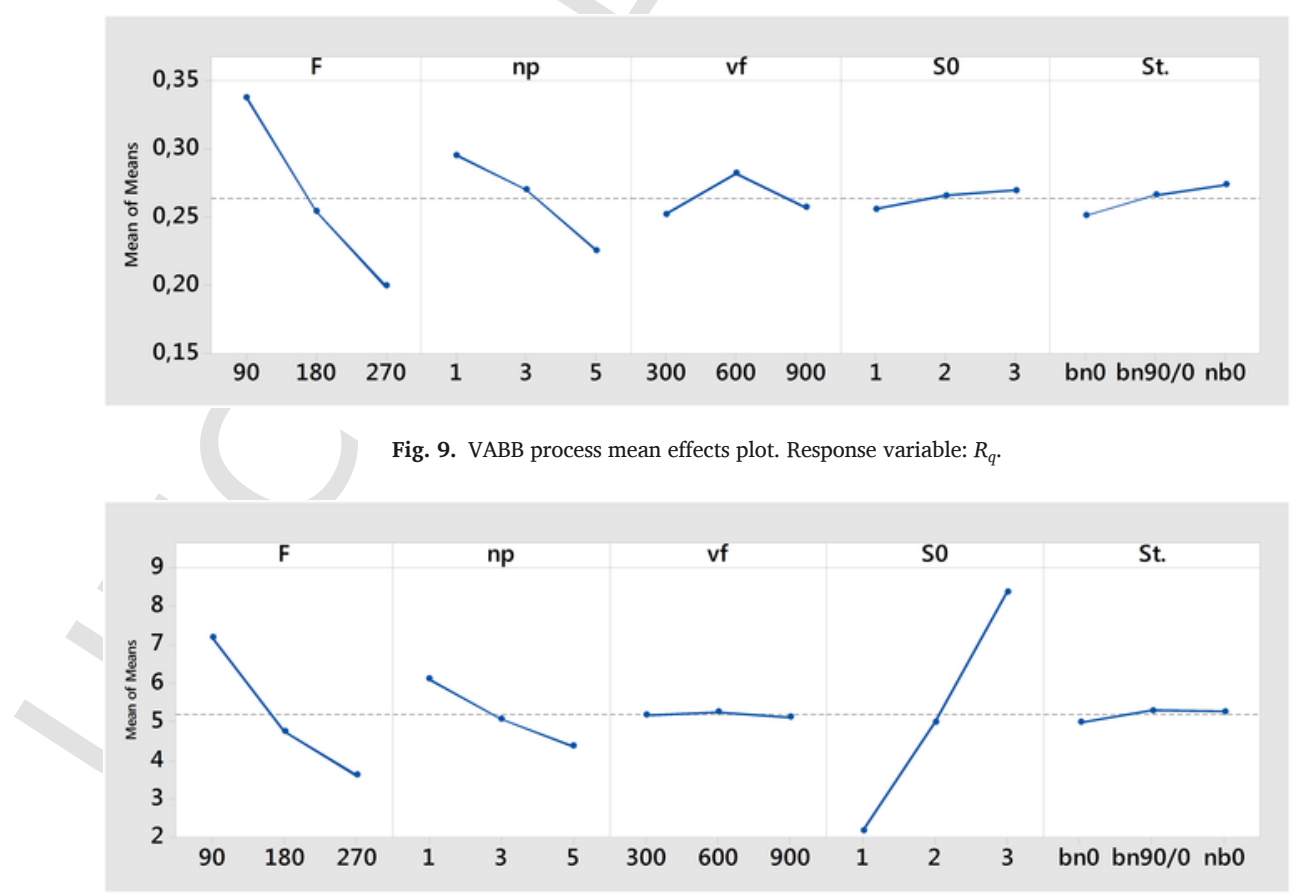

Fig. 10. VABB process mean effects plot. Response variable: $S_{q}$. 


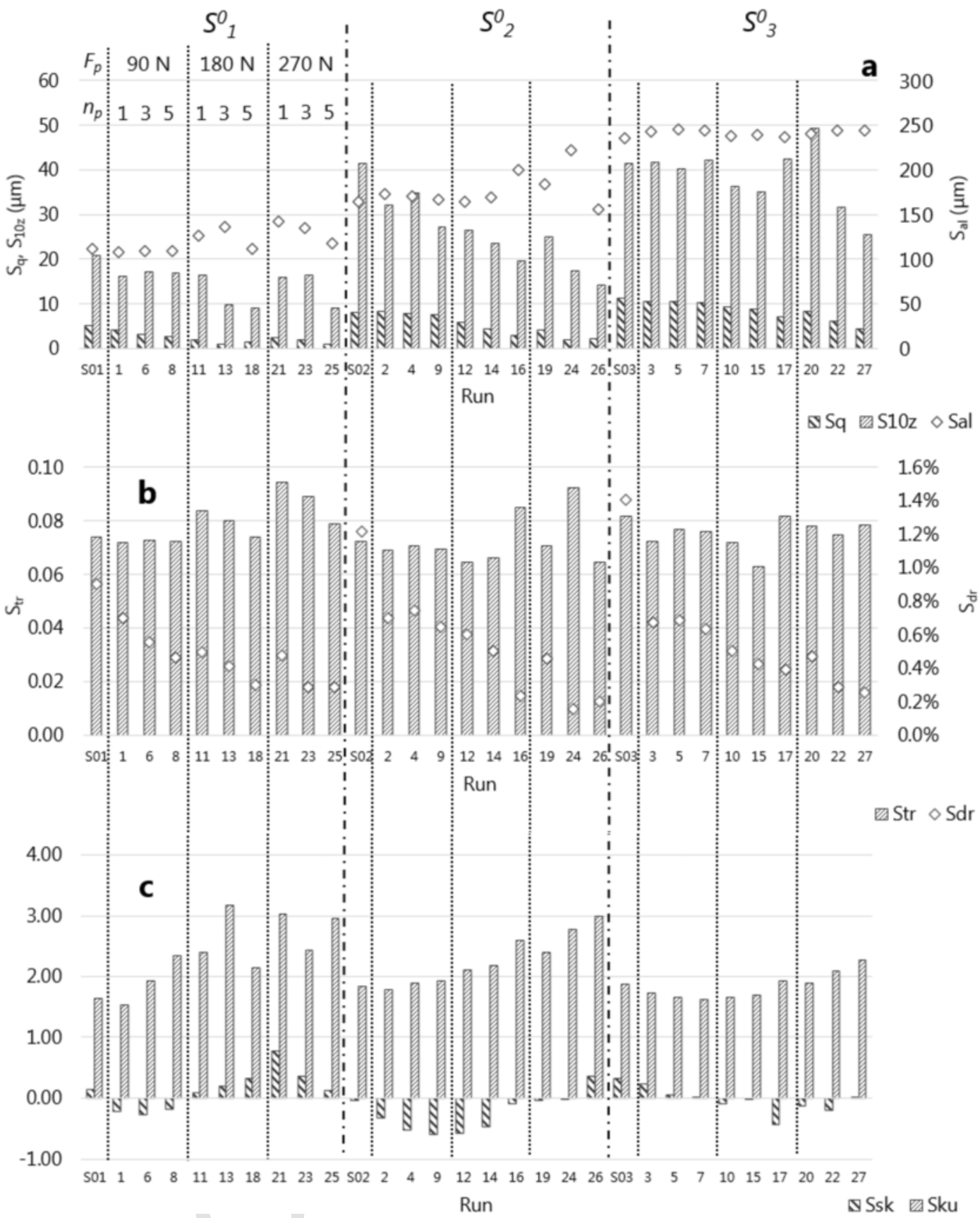

Fig. 11. S parameters describing the topographies of the VABB patches.

the initial texture, as the degree of plastic strain increases with the increase in the preload and number of passes.

The behavior of $S_{q}$ and $S_{10 z}$ parameters is common to all initial surfaces, as a general decrease of both parameters is detected as the preload and number of passes increase simultaneously (Fig. 11a). The only exception of that diminishing trend is present when switching from the $180 \mathrm{~N}-5$ passes test to the $270 \mathrm{~N}-1$ passes test, as a remarkable $S_{q}$ and $S_{10 z}$ increase are detected. This is due to the fact that rising from $180 \mathrm{~N}$ to $270 \mathrm{~N}$ is not enough to compensate the decrease in the number of passes from 5 to 1 . Thus the lower degree achieved albeit the higher force.

From the point of view of these amplitude parameters, the $S^{0}{ }_{1}$ is the surface that shows the best texture results on the whole. In terms of optimal parameters for the tested burnishing conditions, the best $S^{0}{ }_{1}$ surface improvement is attained by applying $180 \mathrm{~N}$ and 5 passes. Increas- ing plastic deformation leads to a surface harm, although at $270 \mathrm{~N}$ and 5 passes, a similar texture results are obtained, but obviously, consuming a larger amount of time. On the contrary, rougher $\mathrm{S}_{2}{ }_{2}$ and $\mathrm{S}_{3}{ }_{3}$ surfaces admit burnishing with the highest force and number of passes to achieve the biggest amplitude reduction. This is due to the fact that burnishing is applied on departing rougher surfaces, and, therefore, a higher force and more number of passes are required to decrease it. The $S^{0}{ }_{1}$ surface starts from an almost superfinished surface, and, therefore, excessive plastic strain can lead to surface harm.

\subsubsection{Spatial and hybrid parameters}

The $S_{d r}$ hybrid parameter reveals a similar decreasing trend with the increasing level of plastic deformation, which proves that higher deformation leads the surface to a more finished state. On the contrary, both spatial parameters $S_{t r}$ and $S_{a l}$ show little variation with the in- 
crease of preload and number of passes (Fig. 11a and b). The former shows in all cases values below 0.1, proving that burnishing does not correct the initial anisotropy shown by the machined surface, and can even intensify it in some cases. This result can be ratified by visual inspection and comparison of the surface before burnishing and after burnishing. Fig. 12 shows both the initial $\mathrm{S}_{3}^{0}$ surface, and its state after VABB with $270 \mathrm{~N}$ and 5 passes (test \#27). The reader shall notice that the initial finishing marks caused by the end-milling process performed with the nose of the hemispherical tool, are unaltered after VABB, while the recipient of plastic deformation is the material that forms the peaks. Therefore, the fact that the machining marks remained unaltered after VABB in most of the tests justifies the little effect of the process on the spatial parameters.

The $S_{t d}$ parameters reveals more interesting information, as it is kept at 90 degrees after testing, except for those tests performed on the $S_{1}^{0}$ surface with $270 \mathrm{~N}$ (tests \#21, \#23 and \#25), in which it takes the value 0 degrees. Consequently, besides reducing the surface scale features, VABB is also able to change the directionality of the main superficial features derived from the previous machining if they are originally of a low scale, and are sufficiently deformed by a high force. This inversion can be conveniently observed in Fig. 8 .

\subsubsection{Amplitude parameters skewness and kurtosis: height distribution}

Skewness and kurtosis are related to the statistical representation of the distribution of heights on the surface. Being amplitude parameters, they are both calculated from the height distribution of the surface, providing information about a possible bias towards negative or positive values (skewness), and whether it is associated to a smoother or an abrupt surface (kurtosis). The reference values for both factors are linked to a Gaussian distribution of heights, described by $S_{s k}=0$ and $S_{k u}=3$. Gaussian surfaces provide the best equilibrium between fluid retention, debris evacuation with minimum wear (highest core material), and are therefore taken as a reference [37]. Indeed, abundant abrupt peaks (low kurtosis, or leptokurtic surfaces) lead to a good lubrication performance, but are due to suffer more wear. The opposite extreme, where surfaces are flat (high kurtosis or platykurtic), are due to suffer less wear, but will not be subject to efficient lubrication. That might have consequences on an increase of working temperature and harm of the surface due to thermomechanical processes such as fretting fatigue.

Again, a differentiation is observed between initial surfaces. $S_{2}^{0}$ and $\mathrm{S}^{0}{ }_{3}$ show a similar trend, as kurtosis increases uniformly in both cases as the degree of plastic strain increases, with exception of the step from $180 \mathrm{~N}-5$ passes to $270 \mathrm{~N}-1$ pass, as already observed. In both cases, $S_{k u}$ increases towards a value of 3 . This value is achieved at $S^{0}$, but not on $\mathrm{S}_{3}^{0}$. On the other hand, the evolution of skewness proves that the first burnishing passes bias the height distribution towards positive values, whereas further plastic strain balances the surface once again, as it reaches value zero.

$S^{0}{ }_{1}$ exhibits a different behavior. Kurtosis reaches a value of 3 for test $180 \mathrm{~N}-3$ passes, then falls once again when increasing to 5 passes. Its behavior is irregular in the following tests, but reaches 3 once again at essays \#21 and \#25, although the former is clearly positively skewed. The behavior of skewness can be similarly interpreted as for the other initial textures, as it decreases at first, whereas a value next to zero is achieved when burnishing with $180 \mathrm{~N}$-3passes. Then, it is unbalanced, and decreases once again to a minimum value at $270 \mathrm{~N}-5$ passes.

Globally, the evolution of all the amplitude parameters lead to similar conclusions, and allow to identify a set of optimal preload-passes couple to burnish the three surfaces and generate a Gaussian surface with minimal RMS with respect to the average center plane. Table 3 summarizes the analyzed results.

Kurtosis is typically assumed as a descriptor of the surface abruptness. To identify this analysis with the VABB results, and further understand the actual nature of kurtosis and skewness evolution, Fig. 13 includes the histogram evolution of heights after burnishing, from the initial surface state, along with the best burnishing conditions for each surface shown at Table 3 , and another singular surface state for the sake of comparison. The results discussed above in this subsection are confirmed, as quasi-Gaussian distributions are exhibited at the histograms corresponding to the best VABB parameters to treat surfaces $\mathrm{S}_{1}^{0}$ and $\mathrm{S}_{2}$. The optimal test \#27 performed at $\mathrm{S}_{3}^{0}$ suggests that the surface could still accept a higher force to increase the kurtosis without biasing the skewness.

Although skewness values are easily identified with the histogram shape - positive values are depicted by the concentration of heights at the negative side, and vice versa - interpreting the kurtosis values is not trivial. Indeed, no purely platykurtic or leptokurtic distributions can be seen at the depicted histograms. The answer lays in the fact that kurtosis is actually a measurement of the relative weight of the tails with regards to the distribution central areas. As Darlington (1970) [38] points outs, kurtosis can be also associated to bimodality, which is the case of burnishing surfaces far from the ideal finishing state (for instance, test \#27 with $S_{k u}=2.266$, represented in Fig. 13). Therefore, in this case, the evolution of kurtosis describes the evolution from multimodal height distributions to unimodal ones, which are, furthermore, unskewed.

\subsection{V parameters and bearing area}

The V parameters are analyzed in this section to judge whether they can complement the information provided by the $\mathrm{S}$ amplitude parameters. Fig. 14a shows the volumetric material values of the burnished surfaces. It is remarkable that both core parameters, $V_{v c}$ and $V_{m c}$, evolve consistently with the $S_{q}$ parameter, accounting for the same scale reduction explained in Section 3.3.1.

Now, to relate both extreme volumetric parameters, $V_{m p}$ and $V_{v v}$, with an evolution in the surface as the degree of plastic deformation increases is not so direct, due to a lack of trend or clear. This observation can be originated in the way they are defined, as they are secondary values calculated from the definition sight of this observation. Consistently, of all three parameters describing the bearing area curves (Fig. $14 \mathrm{~b})$, only $S_{k}$ is due to reveal reliable information. Indeed, it decreases

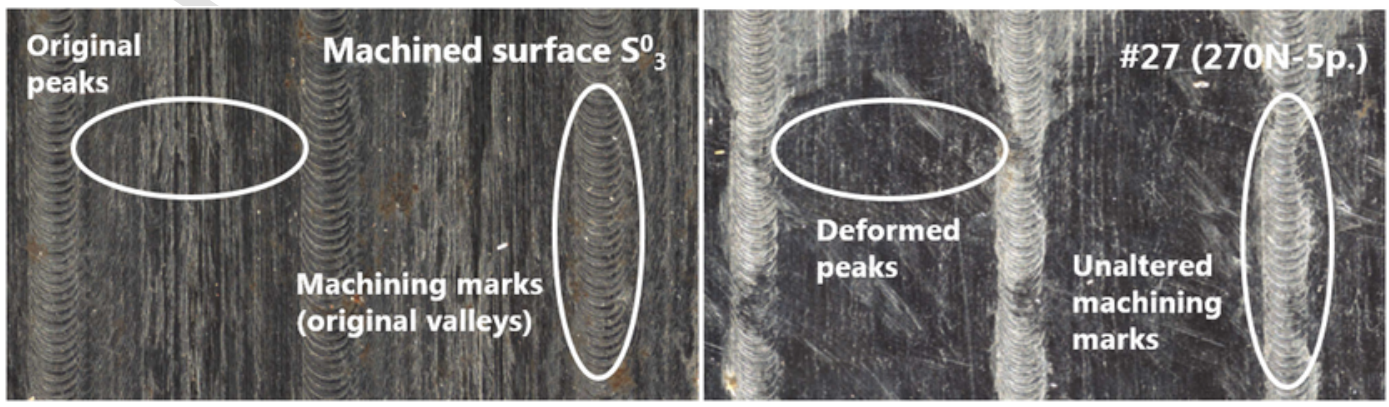

Fig. 12. Optical image of the $\mathrm{S}_{3}^{0}$ surface and that surface after VABB with $270 \mathrm{~N}$ and 5 passes. 
Table 3

Burnishing parameters found to generate Gaussian surfaces with minimum texture amplitudes.

\begin{tabular}{lllll}
\hline $\begin{array}{l}\text { Initial } \\
\text { surface }\end{array}$ & $\begin{array}{l}\text { Characteristic } \\
\text { description }\end{array}$ & $\begin{array}{l}\text { Similar ISO } \\
\text { roughness } \\
\text { grade }\end{array}$ & $\begin{array}{l}F_{p} \\
(\mathrm{~N})\end{array}$ & $n_{p}$ \\
\hline $\mathrm{S}^{0}{ }_{1}$ & $\begin{array}{l}\text { Nearly superfinished } \\
\text { surface }\end{array}$ & $\mathrm{N} 4$ & 180 & 3 \\
$\mathrm{~S}^{0}{ }_{2}$ & $\begin{array}{l}\text { Balanced finished } \\
\text { surface }\end{array}$ & $\mathrm{N} 4-\mathrm{N} 5$ & 270 & 3 \\
$\mathrm{~S}^{0}{ }_{3}$ & $\begin{array}{l}\text { Finished surface, } \\
\text { slightly positively } \\
\text { skewed }\end{array}$ & $\mathrm{N} 5$ & 270 & 5 \\
\hline
\end{tabular}

with $S_{q}$, and, therefore, exemplifies the reduction in scale, as the volumetric core parameters. Consequently, it can be deduced that this set of parameters gives similar information as the S-parameter set, and can be therefore alternatively used.

To understand the misrepresentation of the VABB surfaces by $V_{m p}$, $V_{v v}, S_{v k}$ and $S_{p k}$, parameters, the whole Abbott-Firestone curves are considered. Fig. 15 represents the bearing area curves for all initial surfaces, along with the singular burnishing tests defined at Fig. 13. The highest improvement delivered by VABB at $S^{0}{ }_{1}$ (test \#13) is represented in Fig. 15 a by a flattening effect on the Abbott-Firestone curve. The ulterior harm of it when the preload is increased (test \#21) is depicted by the fact that the curve rebounds back towards the initial state. On the other hand, $S^{0}{ }_{2}$ (Fig. 15b) and $S^{0}{ }_{3}$ (Fig. 15c) exhibit a different behavior, as the bearing area curves become flatter as plastic strain increases, and no harmful effect is observed.

The Gaussian nature of the height distribution, analyzed above through the kurtosis and skewness parameters, is also represented at the bearing area curve. Zero skewness (unbiased height distributions) is associated to curves that cross the $x$ axis at the middle point (50\%). It can be easily observed at a zoomed area of Fig. 15c. For instance, test \#17, negatively skewed, is represented by a bearing area curve which intersect the $x$ axis at $55 \%$.

The platykurtic condition of the burnishing imprints can also be identified in the Abbott-Firestone curves, if it is interpreted as an indicator of bimodality. As the preload and number of passes increase towards the optimal value, the texture evolves towards a Gaussian value of kurtosis. That is revealed by quasi flatness of the surface at the central points, with a smooth slope decrease from the extreme points. Bimodality $\left(S_{k u}<3\right)$ typically exhibited at intermediate states can be seen by abrupt changes of slope in the Abbott-Firestone curves, as has been pointed out it Fig. 15a and b.

The abrupt slope change at the bearing area curves can explain the lack of uniformity observed at the descriptors of the Abbott-Firestone curve described at the beginning of this section. As the parameters are automatically calculated by the intersection of the axis with a line, the descriptors $V_{m p}, V_{v v}, S_{v k}$ and $S_{p k}$ descriptors of the Abbott-Firestone curve should be considered cautiously to describe the results of ball burnishing in states far from the optimal situation. On the other hand, $V_{m c}, V_{v c}$, $S_{k}$ have proven to deliver similar information as the $S_{q}$ amplitude parameters, and can therefore be considered as an alternative set of descriptors of the effects of VABB, when a tribological approach wants to be adopted.

\section{Comparison with the NVABB process}

All ball burnishing conditions included in the experimental array have been also tested under the non-assisted scenario, to establish a comparison point. The topographical features have been measured following the same protocol described above. These results are also digitally available to be consulted. In general, the qualitative behaviors and trends explained for the VABB process are very similar for the NVABB process. Also especially remarkable is the different behavior shown by the $\mathrm{S}^{0}{ }_{1}$ surface with regards to the other two initial machined textures.

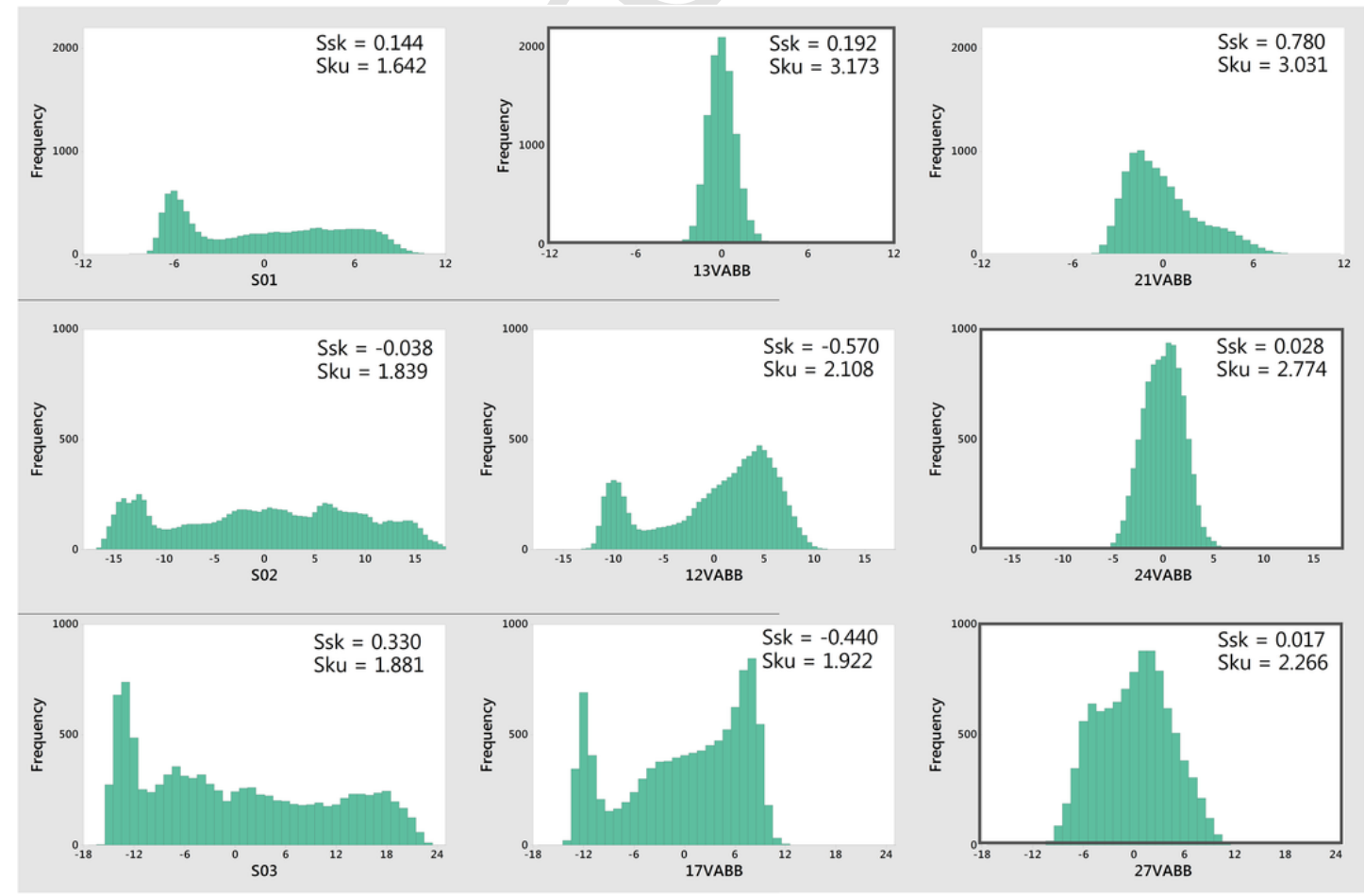

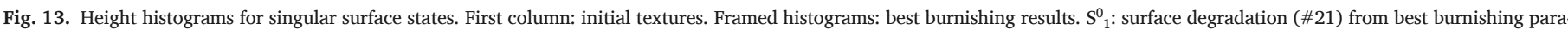
meters (\#13). $\mathrm{S}^{0}$ and $\mathrm{S}^{0}$ : best conditions (\#24 and \#27) attained after intermediate states \#12 and \#17. 


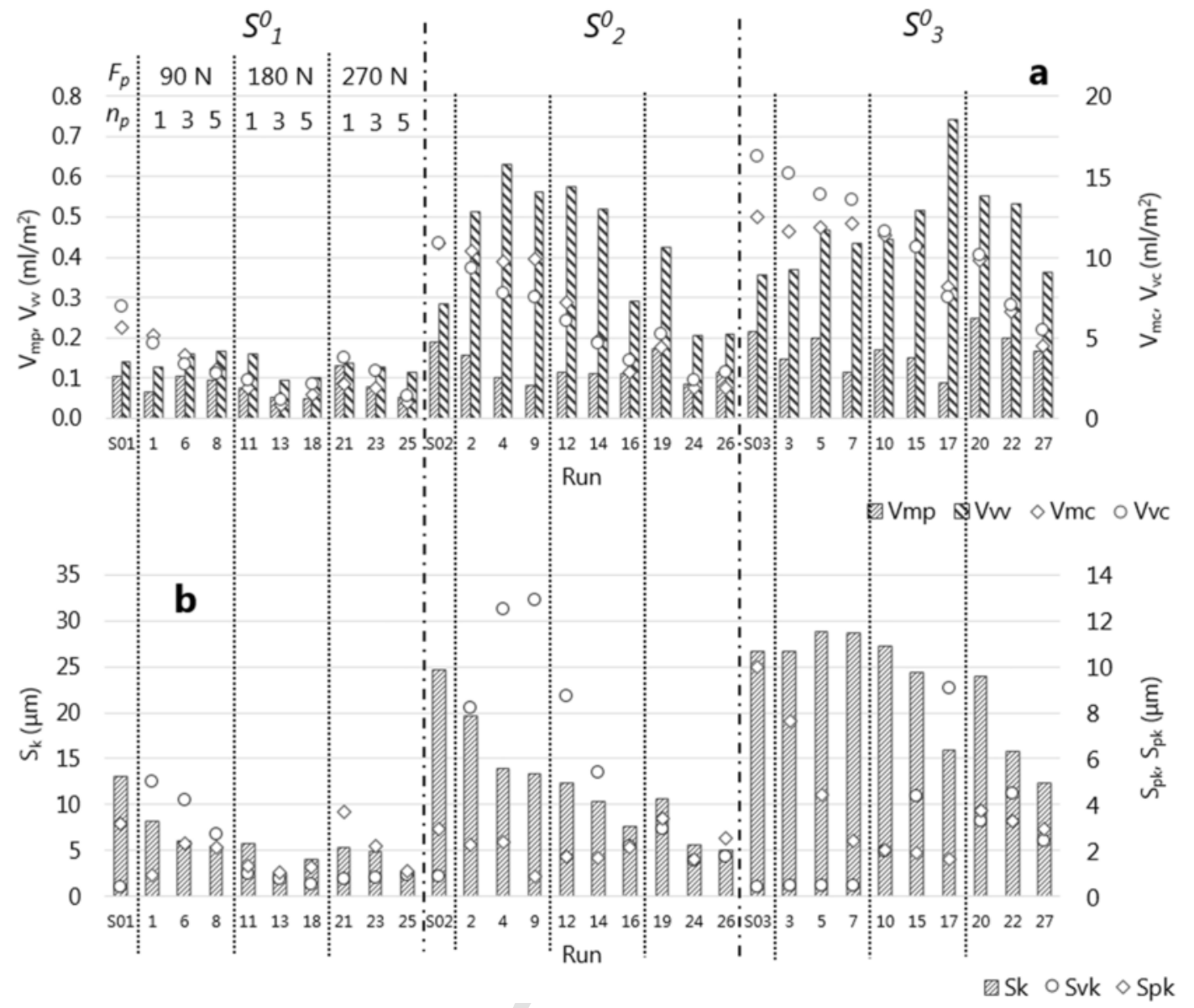

Fig. 14. V parameters describing the topographies of VABB patches. a. Volumetric parameters. b. Bearing area curve descriptors.
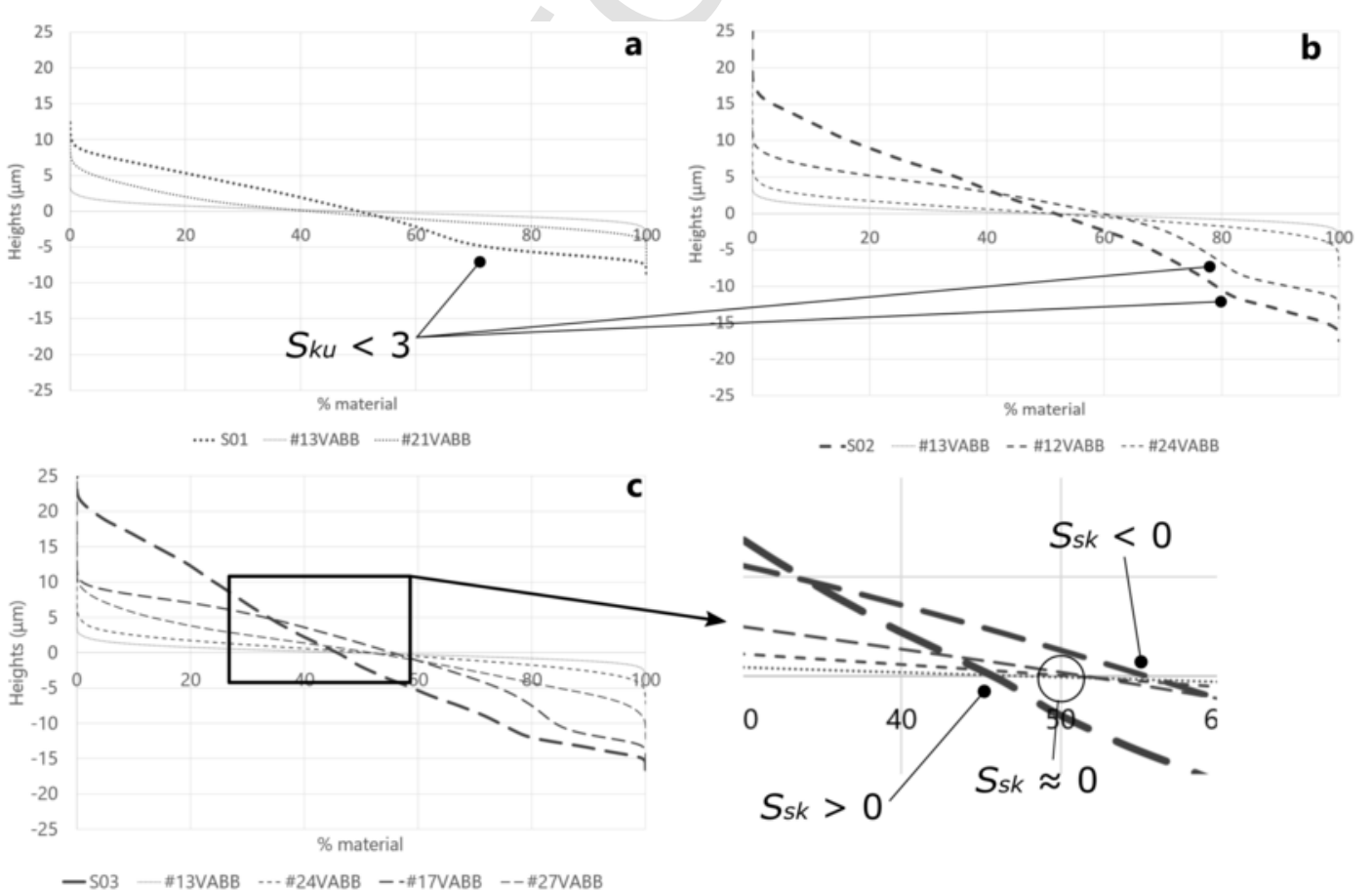

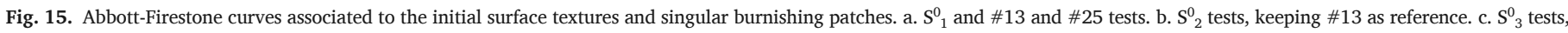
keeping \#13 and \#24 as references. 


\section{1. $R_{q}$ and $S_{q}$ amplitude parameters}

The direct comparison of the general amplitude parameters $S_{q}$ and $R_{q}$ exhibits that they are slightly lower for the NVABB process. Fig. 16a and $\mathrm{b}$ exhibit the mean effects plots taking both parameters as the re- sponse variables. The direct comparison with Figs. 9 and 10 respectively confirms that observation for $S_{q}$, whereas the NVABB shows a higher mean effect in terms of $R_{q}$. This shows that there is a scale effect caused by the VABB process, which affects more intensely at a roughness scale, whereas it is not so effective in reducing the amplitude at a texture level. That can be caused by the fact that acoustoplasticity leads to excessive deformation during the VABB process, and, there-
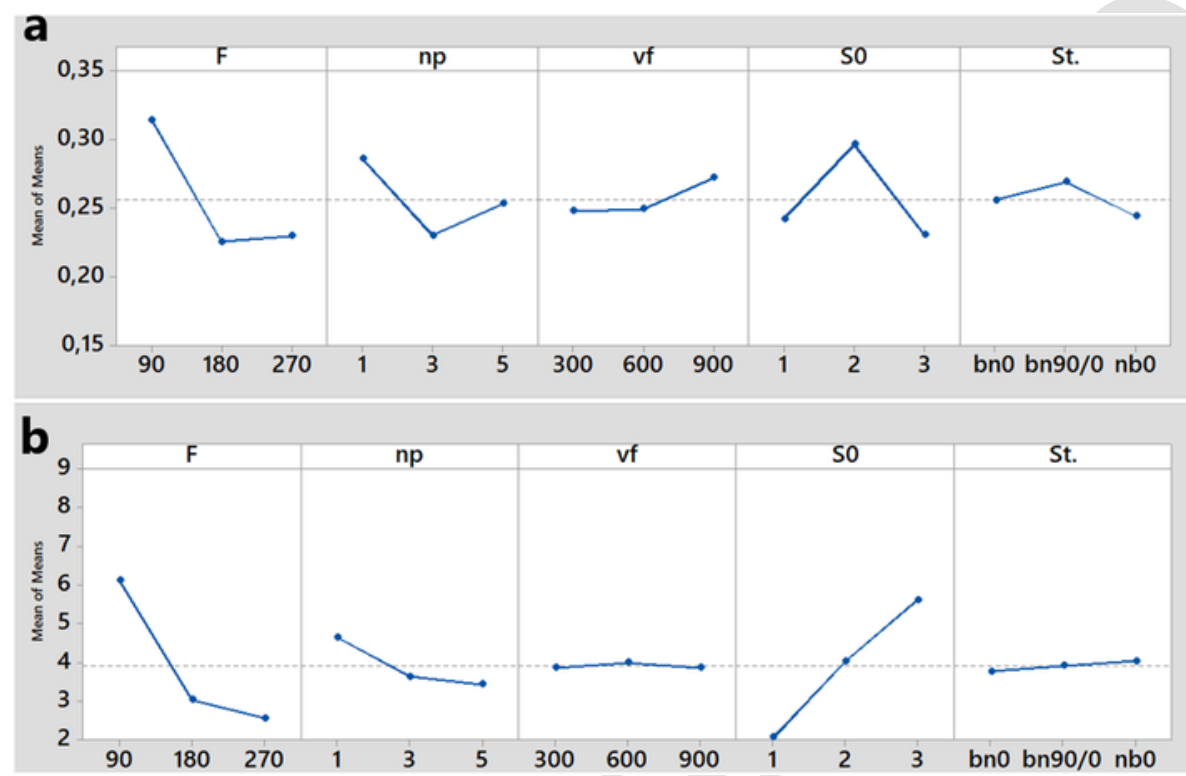

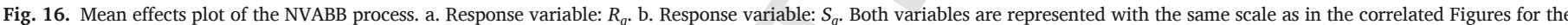
VABB process.

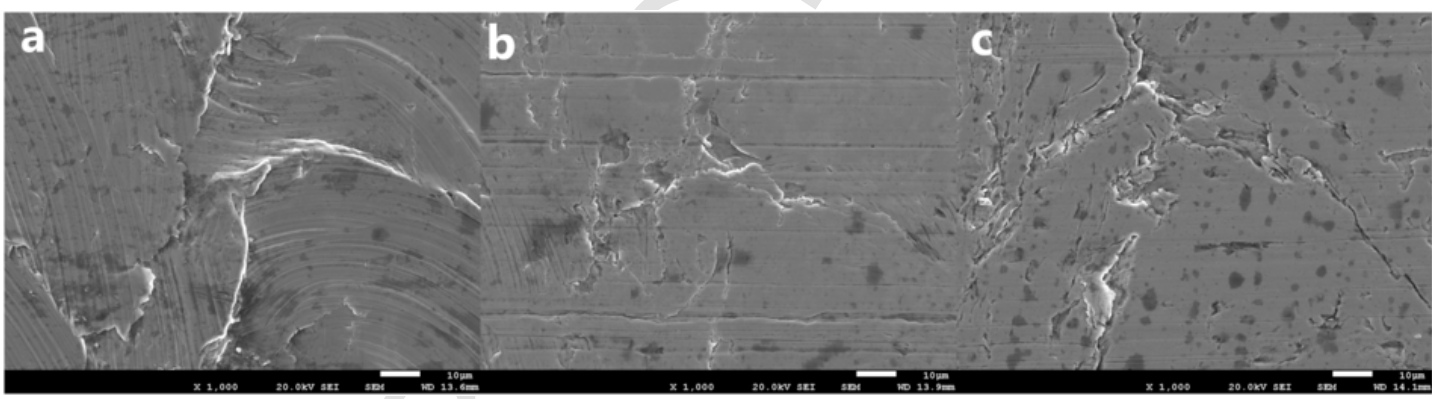

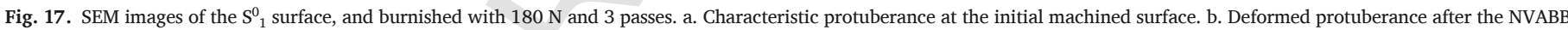
process, with visible longitudinal scratches c. Unscathed surface after the VABB process.

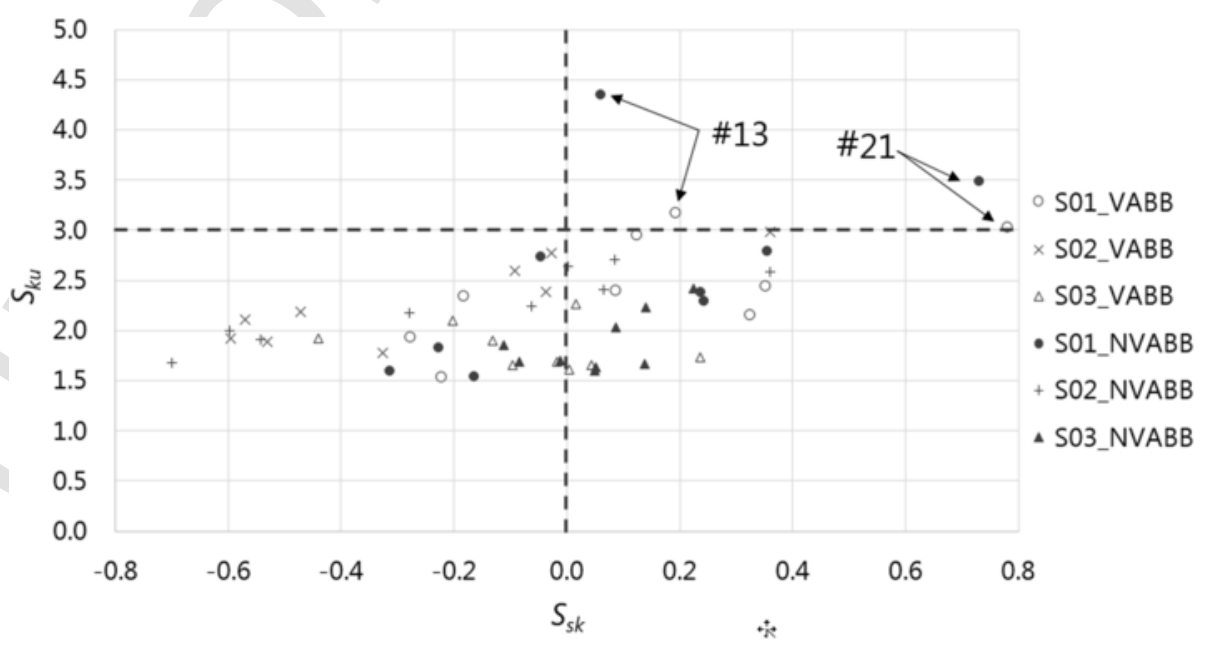

Fig. 18. Skewness and kurtosis amplitude parameters represented for all VABB and NVABB tests. 


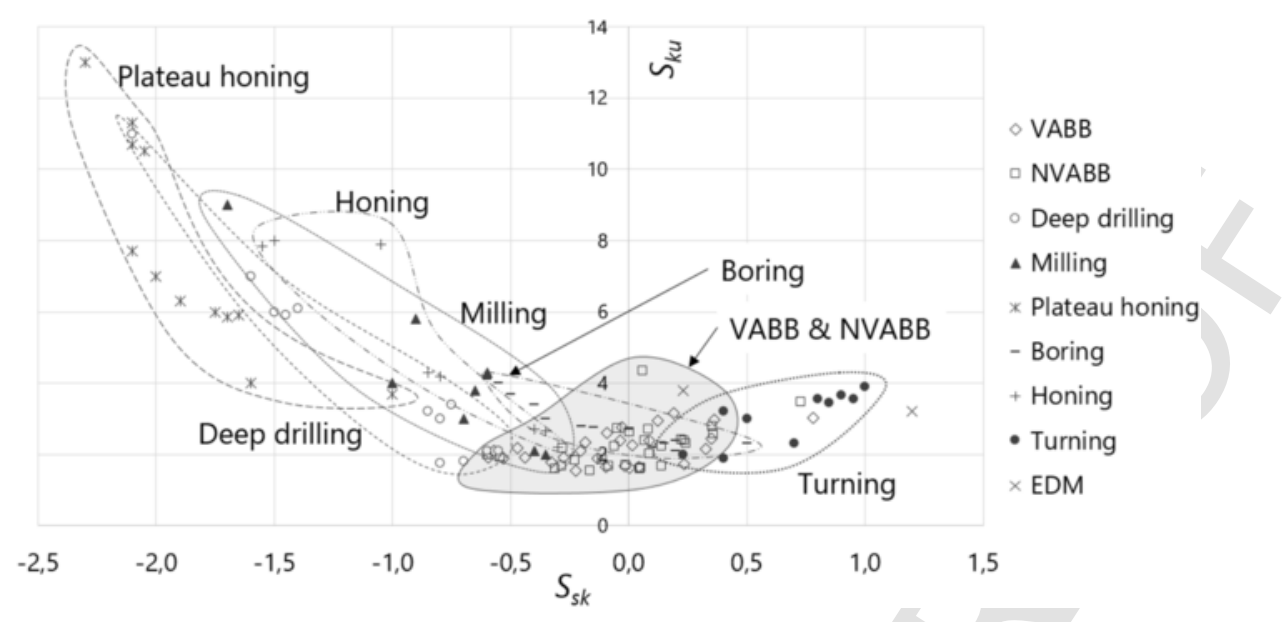

Fig. 19. Position of the VABB and NVABB process with respect to other finishing process. After data found at Griffiths (1986) [39].

fore, the displaced material can generate new irregularities in the surface. All in all, the best burnishing conditions found for the VABB process are the same ones as for NVABB.

A qualitative analysis has also been performed, by comparing the surface state by SEM observation. The initial machined surface shows a typical repetitive pattern caused by the plastic deformation of the remaining material on the surface. Fig. 17a shows a detail of that pattern, which appears to be completely deformed after VABB and NVABB (Fig. 17b and c). Although the surface appears to be equally affected by both processes, NVABB seems to lead to characteristic scratches on the material along the burnishing sense. These scratches can be eventually the source of crack nucleation during in-service functioning. At sight of the absence of these marks on the VABB process, the vibration assisted process can be presented as a more convenient operation to finish an unharmed surface with lower roughness, albeit its slightly higher texture amplitude parameters.

\section{2. $S_{s k}$ and $S_{k u}$ amplitude parameters}

To compare skewness and kurtosis results after VABB and NVABB, all tests have been represented in the Cartesian plane (Fig. 18). This technique can help to characterize the nature of surfaces after finishing through a certain process, as explained by Griffiths (1986) [39]. All results prove to be contained inside a tear-shaped enclosed area. Therefore, both processes are similar in terms of the surface final heights distribution, although the concentration of VABB tests at negative skewness positions shows that NVABB is able to generate less skewed surfaces. This is not an important fact, as, applying the optimal burnishing parameters, both processes lead to Gaussian surfaces. In the same graphic, two outliers can be observed: tested conditions \#13 and \#21. The first set of burnishing parameters executed by the NVABB shows a high kurtosis, whereas the second ones show an especially positive-skewed surfaces.

This approach enables to identify surfaces generated by certain manufacturing process with specific areas of the $S_{s k}-S_{k u}$ plane. It can also be used to compare VABB and NVABB results with other finishing processes (Fig. 19). Firstly, NVABB and VABB show the unique capability of generating both positive and negatively skewed surfaces, as well as unskewed ones, whereas other processes lead to clearly negatively skewed (e.g. honing) or positively skewed surfaces (e.g. turning). Furthermore, ball burnished surfaces can be characterized by low kurtosis values. Although the Gaussian value 3 can be attained with the correct application of parameters, burnishing shows that it generates platykurtic surfaces. As already discussed, this condition comes from the fact that burnished surfaces are associated to bimodal height distributions. That bimodality is corrected as the best combination of preload and number of passes is attained.

\section{Conclusions}

The experimental results of VABB tests through a Taguchi DOE on AISI 1038 ball-end milled surfaces have led to the main conclusion that the process has a triple topological effect on the target surfaces:

- Scale reduction effect, as the irregularities of the target surface topography are successfully reduced, represented by a minimization of the $S_{q}$ parameter.

- Redistribution effect, as the heights of surfaces after the treatment respond to a Gaussian or a quasi-Gaussian distribution, regardless of the excerpted preload $\left(S_{s k}=0\right.$ and $S_{k u}=3$ ). Ball burnishing erases the bimodal height distributed surfaces obtained originally by ball end milling, which is also observed in the regularization of the slope at the Abbott-Firestone curves

- Spatial effect, as the texture anisotropy of the surface, originally defined by the direction of the ball-end milling marks, can be reoriented is enough force is exerted on the surface (change in $S_{t d}$ parameter).

The optimal burnishing parameters inside the explored intervals for each factor are essentially different depending on the initial surface, and the material (the lower its hardness, the higher the effectiveness of the VABB process). For almost super-finished surfaces, an excessive application of preload and number of passes (i.e. level of plastic strain) can lead to harm the surface, whereas less smooth finished surfaces require higher plastic strain to reach the best results.

The comparative results of the process with equivalent NVABB tests shows that, although VABB and NVABB have similar quantitative impact on the surfaces, the surfaces treated by the latter exhibit scratches and surface defects which can be eventually harmful in terms of dynamic behavior of burnished engineering parts, as stress concentrators. Thus the positive effect of adding vibration-assistance to the process.

These conclusions allow to confirm that the VABB process is a feasible and inexpensive alternative to obtain Gaussian surfaces next to super-finishing levels, while it enhances the bearing capacity of the target surfaces. Whereas the NVABB process leads to similar amplitude results and height distribution, it harms the surface, which makes it inadequate to finish parts subjected to in-work fatigue regimes. Moreover, this work also provides a new way to analyze surface texture and topology considering a holistic and wide set of significant descriptors. Further research will complete the surface integrity analysis of the VABB process in terms of hardness, residual stress and influence on the fatigue behavior of AISI 1038 parts. 


\section{Acknowledgements}

This study was financially supported by the Spanish Ministry of Economy and Competitiveness through grant DPI2015-69803-R, and is greatly appreciated.

\section{Appendix A. Supplementary data}

Supplementary data to this article can be found online at https:// doi.org/10.1016/j.surfcoat.2018.05.061.

\section{References}

[1] F.J. Shiou, C.H. Chen, Freeform surface finish of plastic injection mold by using ball-burnishing process, J. Mater. Process. Technol. 140 (1) (2003) 248-254.

[2] F.J. Shiou, C.H. Cheng, Ultra-precision surface finish of NAK80 mould tool steel using sequential ball burnishing and ball polishing processes, J. Mater. Process. Technol. 201 (1) (2008) 554-559.

[3] J.A. Travieso-Rodríguez, G. Dessein, H.A. González-Rojas, Improving the surface finish of concave and convex surfaces using a ball burnishing process, Mater. Manuf. Process. 26 (12) (2011) 1494-1502.

[4] N.H. Loh, S.C. Tam, S. Miyazawa, Surface hardening by ball burnishing, Tribol. Int. 23 (6) (1990) 413-417.

[5] R. Avilés, J. Albizuri, A. Rodríguez, L.N. Lopez De Lacalle, Influence of low-plasticity ball burnishing on the high-cycle fatigue strength of medium carbon AISI 1045 steel, Int. J. Fatigue 55 (2013) 230-244.

[6] A. Rodríguez, L.N. Lopez de Lacalle, A. Celaya, A. Lamikiz, J. Albizuri, Surface improvement of shafts by the deep ball-burnishing technique, Surf. Coat. Technol. 206 (11) (2012) 2817-2824.

[7] A.M. Abrão, B. Denkena, J. Köhler, B. Breidenstein, T. Mörke, The influence of deep rolling on the surface integrity of AISI 1060 high carbon steel, Proc. CIRP 13 (2014) 31-36.

[8] L.N. Lopez de Lacalle, A. Lamikiz, J.A. Sanchez, J.L. Arana, The effect of ball burnishing on heat-treated steel and Inconel 718 milled surfaces, Int. J. Manuf. Technol. 32 (9-10) (2007) 958-968.

[9] A.M. Hassan, A.M. Maqableh, The effects of initial burnishing parameters on non-ferrous components, J. Mater. Process. Technol. 102 (1) (2000) 115-121.

[10] N.H. Loh, S.C. Tam, Effects of ball burnishing parameters on surface finish-a literature survey and discussion, Precis. Eng. 10 (4) (1988) 215-220.

[11] L.N. López de Lacalle, A. Rodriguez, A. Lamikiz, A. Celaya, R. Alberdi, Five-axis machining and burnishing of complex parts for the improvement of surface roughness, Mater. Manuf. Process. 26 (8) (2011) 997-1003.

[12] W. Bouzid, O. Tsoumarev, K. Sai, An investigation of surface roughness of burnished AISI 1042 steel, Int. J. Adv. Manuf. Technol. 24 (1-2) (2004) 120-125.

[13] I. Sherrington, E.H. Smith, Parameters for characterizing the surface topography of engineering components, Proc. Inst. Mech. Eng. C J. Mech. 201 (4) (1987) 297-306.

[14] X.J. Jiang, D.J. Whitehouse, Technological shifts in surface metrology, CIRP Ann Manuf. Technol. 61 (2) (2012) 815-836.

[15] K.W. Siu, A.H.W. Ngan, I.P. Jones, New insight on acoustoplasticity-ultrasonic irradiation enhances subgrain formation during deformation, Int. J. Plast. 27 (5) (2011) 788-800.

[16] O. Izumi, K. Oyama, Y. Suzuki, Effects of superimposed ultrasonic vibration on compressive deformation of metals, T. Jpn. I. Met. 7 (3) (1966) 162-167.
[17] H.O.K. Kirchner, W.K. Kromp, F.B. Prinz, P. Trimmel, Plastic deformation under simultaneous cyclic and unidirectional loading at low and ultrasonic frequencies, Mater. Sci. Eng. (2) (1985) 197-206.

[18] S.S. Pande, S.M. Patel, Investigations on vibratory burnishing process, Int. J. Mach. Tool Manuf. D. R. 24 (3) (1984) 195-206.

[19] G. Gomez-Gras, J.A. Travieso-Rodriguez, H.A. González-Rojas, A. Nápoles-Alberro, F.J. Carrillo, G. Dessein, Study of a ball-burnishing vibration-assisted process, Proc. Inst. Mech. Eng. B J. Eng. 229 (1) (2015) 172-177.

[20] J.A. Travieso-Rodriguez, G. Gomez-Gras, G. Dessein, F. Carrillo, J. Alexis, J. Jorba-Peiro, N. Aubazac, Effects of a ball-burnishing process assisted by vibrations in G10380 steel specimens, Int. J. Adv. Manuf. Technol. 81 (9-12) (2015) $1757-1765$

[21] J.A. Travieso-Rodríguez, G. Gomez-Gras, J.J. Peiró, F. Carrillo, G. Dessein, J. Alexis, H. Gonzalez-Rojas, Experimental study on the mechanical effects of the vibration-assisted ball-burnishing process, Mater. Manuf. Process. 30 (12) (2015) 1490-1497.

[22] A.T. Bozdana, N.N. Gindy, H. Li, Deep cold rolling with ultrasonic vibrations-a new mechanical surface enhancement technique, Int. J. Mach. Tools Manuf. 45 (6) (2005) 713-718.

[23] A.T. Bozdana, N.Z. Gindy, Comparative experimental study on effects of conventional and ultrasonic deep cold rolling processes on Ti6Al4V, Mater. Sci. Technol. 24 (11) (2008) 1378-1384.

[24] J. Huuki, S.V. Laakso, Integrity of surfaces finished with ultrasonic burnishing, Proc. Inst. Mech. Eng. B J. Eng. 227 (1) (2013) 45-53.

[25] J. Huuki, M. Hornborg, J. Juntunen, Influence of ultrasonic burnishing technique on surface quality and change in the dimensions of metal shafts, Hindawi J. Eng. 2014 (2014) https://doi.org/10.1155/2014/124247

[26] R. Jerez Mesa, J.A. Travieso-Rodriguez, G. Gomez-Gras, J. Lluma-Fuentes, Development, characterization and test of an ultrasonic vibration assisted ball burnishing tool, J. Mater. Process. Technol. 257 (2018) 203-212

[27] N.H. Loh, S.C. Tam, S. Miyazawa, Application of experimental design in ball burnishing, Int. J. Mach. Tools Manuf. 33 (6) (1993) 841-852.

[28] C.Y. Seemikeri, P.K. Brahmankar, S.B. Mahagaonkar, Investigations on surface integrity of AISI 1045 using LPB tool, Tribol. Int. 41 (8) (2008) 724-734.

[29] G.D. Revankar, R. Shetty, S.S. Rao, V.N. Gaitonde, Analysis of surface roughness and hardness in ball burnishing of titanium alloy, Measurement 58 (2014) 256-268.

[30] G. Taguchi, S. Chowdhury, Y. Wu, Taguchi's Quality Engineering Handbook, John Wiley \& Sons, New Jersey, 2005

[31] W.P. Dong, P.J. Sullivan, K.J. Stout, Comprehensive study of parameters for characterizing three-dimensional surface topography I: some inherent properties of parameter variation, Wear 159 (2) (1992) 161-171.

[32] W.P. Dong, P.J. Sullivan, K.J. Stout, Comprehensive study of parameters for charac terizing three-dimensional surface topography II: statistical properties of parameter variation, Wear 167 (1) (1993) 9-21.

[33] W.P. Dong, P.J. Sullivan, K.J. Stout, Comprehensive study of parameters for charac terising three-dimensional surface topography: III: parameters for characterising amplitude and some functional properties, Wear 178 (1-2) (1994) 29-43.

[34] W.P. Dong, P.J. Sullivan, K.J. Stout, Comprehensive study of parameters for characterising three-dimensional surface topography: IV: parameters for characterising spatial and hybrid properties, Wear 178 (1-2) (1994) 45-60.

[35] E.J. Abbot, F.A. Firestone, Specifying surface quality, Mech. Eng. 55 (9) (1933) 569-572.

[36] T.G. King, T.A. Spedding, On the relationships between surface profile height parameters, Wear 83 (1) (1982) 91-108.

[37] K.J. Stout, E.J. Davis, Surface topography of cylinder bores-the relationship between manufacture, characterization and function, Wear 95 (2) (1984) 111-125.

[38] R.B. Darlington, Is kurtosis really "peakedness"?, Am. Stat. 24 (2) (1970) 19-22.

[39] B.J. Griffiths, Problems in measuring the topography of machined surfaces produced by plastic deformation mechanisms, Wear 109 (1-4) (1986) 195-205. 